\title{
ON THE NUMBER OF SOLUTIONS TO PLATEAU'S PROBLEM
}

\author{
BY A. J. TROMBA 1
}

Communicated by S. S. Chern, August 28, 1975

Introduction. Since its formulation by Plateau in the 19th century, little (see [2], [4]) has been known about the number of simply connected minimal surfaces spanning a simple closed curve $\Gamma \subset R^{3}$. Existence was proved in the thirties by J. Douglas [1] and T. Radó [5]. In the paragraphs below we indicate how a new topological theory partially describes the way in which the number of minimal surfaces spanning a curve changes as the curve changes.

I. Formulation of the problem. Let $H^{r+2}\left(S^{1}, R^{n}\right)$ be the Sobolev Hilbert space of $H^{r+2}$ maps the unit circle $S^{1}$ into $R^{n}$, with $r \geqslant 5$. Let $A=\operatorname{Emb}\left(S^{1}, R^{3}\right)$ be the open submanifold of $H^{r+2}\left(S^{1}, R^{3}\right)$ which consists of embeddings of $S^{1}$ into $R^{3}$. Let $\Gamma$ be the image of such an embedding $\alpha \in A$. Set $\eta^{\alpha}$ to be the component of $H^{2}\left(S^{1}, \Gamma\right)$ \{ the $C^{r}$ Hilbert manifold of $H^{2}$ maps from $S^{1}$ to $\Gamma$ \} determined by the embedding $\alpha$. Let $M^{\alpha}$ be the open submanifold of $\eta^{\alpha}$ consisting of the diffeomorphisms. For every $u \in H^{2}\left(S^{1}, \Gamma\right) \subset H^{2}\left(S^{1}, R^{3}\right)$ we can extend $u=\left(u_{1}, \ldots, u_{n}\right)$ harmonically to the disc $D$. Define the smooth energy functional $E_{\alpha}: \eta^{\alpha} \rightarrow R$ by

$$
E_{\alpha}(u)=\frac{1}{2} \sum_{i=1}^{3} \int_{D}\left[\left(\frac{\partial u_{i}}{\partial x}\right)^{2}+\left(\frac{\partial u_{i}}{\partial y}\right)^{2}\right] d x d y .
$$

Denote by $\bar{M}^{\alpha}$ the closure of $M^{\alpha}$ in $\eta^{\alpha}$.

J. Douglas showed, in his pioneering work [1], that the critical points of $E_{\alpha}$ in $\bar{M}^{\alpha}$ are simply connected minimal surfaces spanning $\Gamma$. We are interested in obtaining information on the number of critical points of $E_{\alpha}$ on $\bar{M}^{\alpha}$.

II. The theory. Let $M$ be a connected smooth Banach manifold and $K: T^{2} M \rightarrow T M$ a connection map. In [6] the author defines a smooth vector field $X: M \rightarrow T M$ to be Fredholm with respect to $K$ if for each $p \in M$ the covariant derivative of $X$ with respect to $K, \nabla X(p)$, which is a linear map of $T_{p} M$ to itself, is linear Fredholm. By the index of $X$ we mean the dim ker $\nabla X(p)-$ dim coker $\nabla X(p)$. A Fredholm vector field is Palais-Smale if $\nabla X(p)$ is of the form $I+C$, where $C$ is a completely continuous linear map. Palais-Smale vector fields have index zero. 57D25.

AMS (MOS) subject classifications (1970). Primary 35G20, 49F10, 58E15; Secondary

${ }^{1}$ Research partially supported by National Science Foundation grants GP-39060 and MPS72-05055 A02. 
Let $X$ be a Palais-Smale vector field on $M$ with finitely many isolated zeros in the interior of $M$. Then using the degree theory developed in [3] one can define the degree of $X$ at a zero $p$, which we denote by $(\operatorname{deg} X)(p)$. The Euler characteristic $\chi(X)$ is defined to be

$$
\chi(X)=\sum_{p \in \operatorname{zeros}(X)}(\operatorname{deg} X)(p) .
$$

If $X$ has no zeros then $\chi(X)=0$. By using elementary transversality techniques, the Euler characteristic can be defined for Palais-Smale vector fields with a compact set of zeros in the interior of $M$.

\section{Applications.}

THEOREM 1. There exists a smooth connection $K_{\alpha}$ on the second tangent bundle $T^{2} \eta^{\alpha}$ and a smooth vector field $X^{\alpha}: \eta^{\alpha} \rightarrow T \eta^{\alpha}$ which is Palais-Smale with respect to the connection $K_{\alpha}$ and whose zeros are precisely all the critical points of $E_{\alpha}$. Moreover, $X^{\alpha}\left(E_{\alpha}\right)=d E_{\alpha}(u)\left(X^{\alpha}(u)\right) \geqslant 0$.

Definition. Let $u \in \eta^{\alpha}$ be a minimal surface. A branch point $p \in D$ of $u$ is a point where the map $u: D \rightarrow \mathbf{R}^{3}$ fails to be an immersion. An embedding $\alpha \in A$ is fine if all minimal surfaces spanning $\Gamma=\alpha\left(S^{1}\right)$ are free of branch points.

In [4] Radó showed that if $\alpha$ is not "too complicated" then $\alpha$ is fine. In particular, he showed that $\alpha$ is fine if there existed no point $q \in \mathbf{R}^{\mathbf{3}}$ such that every hyperplane through $q$ intersected $\Gamma$ in at least four points.

THEOREM 2. Let $F \subset A$ be the set of fine embeddings. Then $F$ is open in $A$, and hence open in $H^{r+2}\left(S^{1}, \mathbf{R}^{3}\right)$.

Conjecture. $F$ is dense in $A$, or perhaps the open set of curves which admit no minimal surfaces with boundary branch points is dense in $A$.

Let $G$ be the three dimensional noncompact Lie group of bijective holomorphic maps of the disc onto itself. The functional $E_{\alpha}$ and the vector field $X^{\alpha}$ of Theorem 1 will be equivariant with respect to the action of $G$. Therefore, there is no hope that the critical points of $E_{\alpha}$ in $\bar{M}^{\alpha}$ will be isolated since the orbit of any critical point will consist of critical points. Applying general transversality techniques we obtain

THEOREM 3. For an open dense set of embeddings $V \subset F$ the zeros of $X^{\alpha}, \alpha \in V$, in $M^{\alpha}$ are nondegenerate (and therefore isolated) three dimensional submanifolds of $\eta^{\alpha}$. Moreover, for such $\alpha \in V$ there are only finitely many such critical submanifolds.

In general minimal surfaces on the same orbit are identified. Doing this we find

THEOREM 4. If $\alpha \in V$ and $\gamma \in F, \gamma=\alpha+\rho$, is sufficiently close to $\alpha$, then the minimal surfaces spanning $\gamma$ are smooth functions of the parameter $\rho$. 
COROLlary. If $\alpha \in V$ and $\gamma \in F$ is sufficiently close to $\alpha$, then the geometric number of minimal surfaces spanning $\gamma$ is equal to the number spanning $\alpha$.

Corollary. Any curve sufficiently close to a plane curve has a unique minimal surface spanning it.

Let $\gamma$ belong to $F$. We can define the Euler-characteristic of the corresponding vector field $X^{\gamma}$, and we take this to be the definition of the algebraic number of minimal surfaces spanning the image $\gamma\left(S^{1}\right)$.

Applying an Euler-Hopf theorem for Palais-Smale vector fields we get

THEOREM 5. Let $\gamma_{0}$ and $\gamma_{1}$ be fine embeddings. Suppose further that $\gamma_{0}$ is isotopic to $\gamma_{1}$ through a family $\gamma_{t}, 0 \leqslant t \leqslant 1$, of fine embeddings. Then the algebraic number of minimal surfaces spanning $\gamma_{0}$ is equal to the number spanning $\gamma_{1}$.

\section{REFERENCES}

1. J. Douglas, Solution to the problem of Plateau, Trans. Amer. Math. Soc. 33 (1931), 263-321.

2. R. Courant, Dirichlet's principle, conformal mapping, and minimal surfaces, Interscience, New York, 1950. MR 12, 90.

3. K. D. Elworthy and A. J. Tromba, Differentiable structures and Fredholm maps on Banach manifolds, Proc. Sympos. Pure Math., vol. 15, Amer. Math. Soc., Providence, R, I., 1970, pp. 45-94. MR 41 \#9299.

4. T. Rádo, On the problem of Plateau, Ergebnisse der Mathematik und Ihrer Grenzgebiete, Springer-Verlag, Berlin, 1933.

5. - The problem of least area and the problem of Plateau, Math. Z. 32 (1930), 763-796.

6. A. Tromba, Fredholm vector fields and a transversality theorem, J. Functional Analysis (to appear).

7. R. Bohme, Die Zusammenhangskomponenten der Lösungen analytischer Plateauprobleme, Math. 133 (1973), 31-40. MR 49 \#11363.

8. R. Bohme and F. Tomi, Zur Struktur der Lösungsmenge des Plateauproblems, Math. Z. 133 (1973), 1-29. MR 49 \#11362.

9. F. Tomi, On the local uniqueness of the problem of least area, Arch. Rational Mech. Anal. 52 (1973), 312-318. MR 49 \#11364.

SCHOOL OF MATHEMATICS, INSTITUTE FOR ADVANCED STUDY, PRINCETON, NEW JERSEY 08540

DEPARTMENT OF MATHEMATICS, UNIVERSITY OF CALIFORNIA, SANTA CRUZ, CALIFORNIA 95064 